\title{
Statistical Modeling of Disturbed Antennas Based on the Polynomial Chaos Expansion
}

\author{
Jinxin Du, Christophe Roblin, Member, IEEE
}

\begin{abstract}
A new methodology of statistical modeling of the far field (FF) radiated by antennas undergoing random disturbances is presented. Firstly, the radiated FF is transformed into a parsimonious form using the Spherical Modes Expansion Method (SMEM); then a surrogate model relating the parsimonious field with the input random parameters is constructed using the Polynomial Chaos Expansion Method (PCEM). The combination of the SMEM and the PCEM allows to develop a compact and precise model with a minimized experimental design cost. The obtained model is computationally costless for generating statistical samples of disturbed antennas easily usable as surrogate models in various types of analyses. In order to demonstrate its performance, the proposed methodology is validated with a deformable canonical antenna - a dipole undergoing three independent random deformations (stretching, bending and torsion), deriving a compact and precise surrogate model.
\end{abstract}

Index Terms - Antennas, antenna surrogate model, statistical antenna modeling, spherical harmonics, polynomial chaos.

\section{INTRODUCTION}

$\mathrm{N}$ OWADAYS antennas are increasingly used in complex and fluctuating environment where random disturbances from the nearby surroundings can have non-negligible impact on antennas performance. Besides, antennas may be also subject to other strong randomness due to their intrinsic variability. This is the case for wearable, deformable or reconfigurable antennas [1], which are rapidly emerging in the communication and medical fields. Therefore, quantitatively characterize the performance of antennas undergoing random disturbances is of significant importance.

In the past few years, efforts have been made to interpret random effects on antenna global performance indicators (GPIs) such as the total efficiency, bandwidth, resonance frequency, radiation patterns [2][4], etc., or on diffuse channels [5] To our knowledge, it is the first time a methodology is proposed for modeling statistically the FF radiated by antennas in uncertain configurations or environments. Such overall statistical modeling provides a complete and efficient substitute for simulated (with EM solvers) or real antennas: any FF antenna response in the considered fluctuating conditions can be computed with a negligible computational cost. This kind of surrogate model can be useful for various types of analyses beyond

This paper is submitted on April 22, 2016 for review.

J. Du and C. Roblin are with the Department of Communication and Electronics, Télécom ParisTech (Institut Mines-Télécom and LTCI), Paris 75634, France (e-mail: jinxin.du@telecom-paristech.fr).
GPI-related ones, such as combined modeling approaches, e.g. hybrid statistical antenna and directional channel models analyses [6] (joint antenna/channel analyses can be driven with a low computational cost) or complete antenna models plugged in asymptotic simulators (e.g. ray tracing), etc., notably when the knowledge of both polarization and phase is mandatory (e.g. for MIMO systems). The only real drawback of the proposed method compared to the statistical modeling of specific GPIs is an increased complexity of the modeling.

In this letter, we present a methodology for the overall modeling of "variable antennas", in which the procedure is split into two steps. The first one is to achieve a parsimonious but complete representation of the radiated FF, and the second consist in developing a statistical model which can quantify the effects of antennas random variability on their radiation properties. Several techniques such as the Spherical Mode Expansion Method (SMEM) [7], the Singularity expansion method [8], [9], and the Fourier series expansion [10] can be used to efficiently represent the field. The underlying common principle is to represent the EMF in a more suitable space in order to achieve a considerable compression of information, prior to consider randomness effects with an achievable statistical approach. The SMEM was chosen for its efficiency as regards parsimony, in particular for small to moderate size antennas (for terminals, sensors, RFID tags, etc.). The Polynomial Chaos Expansion Method (PCEM) was chosen for its efficiency to model stochastic multivariate input/output problems, and its ability to deduce compact and accurate surrogate models with considerably lower Experimental Design (ED) cost than classical methods such as e.g. Monte Carlo. Once the model is available, one can easily generate antennas corresponding to any values of the random input parameters.

\section{Proposed Methodology}

\section{A. Parsimony of the EMF with the SMEM}

According to [11], the electromagnetic far field of any passive antenna can be fully represented by its Antenna Transfer Function (ATF) $\mathcal{H}(f, \hat{\mathbf{r}})$. Based on the Spherical Modes Expansion theory, the ATF can be expanded as:

$$
\mathcal{H}(f, \hat{\mathbf{r}}) \triangleq \frac{r e^{j k r}}{a_{1}(f)} \sqrt{\frac{4 \pi}{\eta_{0}}} \cdot \mathbf{E}^{\infty}(f, \mathbf{r})=\sum_{p=1}^{\infty} H_{p}(f) \hat{\Psi}_{p}(\hat{\mathbf{r}})
$$

where $p=2\{n(n+1)+m-1\}+s$ is the condensed global index related to the modality $s$ ( $=1$ or 2 ), degree $n$, and order $m$ [7]. In the spherical coordinate system, the Vector Spherical Harmonics (VSHs) $\widehat{\psi}_{p}(\hat{\mathbf{r}})$ are orthonormal bases in the space of vector spherical functions and $H_{p}(f)$ are complex coefficients which are obtained by projection $H_{p}(f)=<\mathcal{H}(f, \hat{\mathbf{r}}), \widehat{\psi}_{p}^{*}(\hat{\mathbf{r}})>$. 
In practice the SME is truncated to a maximal degree $N_{\max }$ (i.e. to $P_{\max }=2 N_{\max }\left(N_{\max }+2\right)$ ) corresponding to the most energetically dominant VSH modes $\left(N_{\max } \approx k r_{0}+\mathcal{O}\left(\sqrt[3]{k r_{0}}\right)\right.$, where $r_{0}$ is the radius of the antenna minimal sphere [12]), which is rather low for small/moderate size antennas. Moreover, as the real and imaginary parts of VSH modes have independent contributions to the total energy, we can keep only most dominant real and/or imaginary parts of $H_{p}(f)$, as in (2):

$$
\mathcal{H}(f, \hat{\mathbf{r}})=j \sum_{q \in I_{d}}(-j)^{\bmod (q, 2)} \mathcal{H}_{q}(f) \hat{\Psi}_{\lceil q / 2\rceil}(\hat{\mathbf{r}})
$$

where $q=2(p-1)+u$ with $u \in\{1,2\}, \mathcal{H}_{q}(f)=\mathfrak{R e}\left\{H_{p}(f)\right\}$ or $\mathcal{H}_{q}(f)=\mathfrak{I m}\left\{H_{p}(f)\right\}$ if $u=1$ or 2 ; $" \bmod (a, n) "$ means " $a$ modulo $n$ " and " $\ulcorner\cdot\rceil$ " is the ceiling round function; $I_{d}$ is the index set of the selected most dominant $\mathcal{H}_{q}(f)$ such that the collected energy reaches a predefined relative threshold $E_{c_{-} t h}($ e.g. $99 \%)$ compared to the total energy corresponding to degree $N_{\max }$. Note that the cardinal of $I_{d}$ is expected to be much smaller than $2 P_{\max }$, achieving a sparser field representation which is beneficial for improving the model's compactness.

By reversing the SME transform we are able to reconstruct the ATF from the set of selected dominant $\mathcal{H}_{q}(f)$. To evaluate the accuracy of the truncated expansion, an error indicator named Relative Root Mean Square Error of the Realized Gain (RRMSE of $G_{r}$ ) is defined in (3):

$$
\operatorname{RRMSE}\left(G_{r}(f)\right)=\frac{\sqrt{\iint_{\Omega_{m}}\left[G_{r}^{\text {init }}(f, \hat{\mathbf{r}})-G_{r}^{r c s}(f, \hat{\mathbf{r}})\right]^{2} d \Omega / \Omega_{m}}}{\iint_{\Omega_{m}} G_{r}^{\text {init }}(f, \hat{\mathbf{r}}) d \Omega / \Omega_{m}}
$$

where $G_{r}^{\text {init }}$ and $G_{r}^{r c s}$ denote respectively the realized gain of the initial and reconstructed fields, $d \Omega$ is the infinitesimal solid angle, $\Omega_{m}=\left\{(\theta, \varphi) \mid G_{r}(\theta, \varphi) \geq \underset{(\theta, \varphi)}{\operatorname{Max}}\left[G_{r}(\theta, \varphi)\right]-10 d B\right\}$ is the main lobe(s) solid angle and reminding that $G_{r}(f, \hat{\mathbf{r}})=\|\mathcal{H}(f, \hat{\mathbf{r}})\|^{2}$ [11]. This indicator is also used to assess the field variations due to disturbances, $G_{r}^{r c s}$ and $G_{r}^{\text {init }}$ being replaced by the $G_{r}$ of the fields with and without disturbances.

\section{B. Polynomial Chaos Expansions (PCE) of VSH coefficients}

The PCE is an efficient method for describing the uncertainty propagation characteristics of an uncertain system. The input/output relation representing such system is denoted $Y=\mathcal{M}(\boldsymbol{X})$, where $\boldsymbol{X}=\left(X_{1}, X_{2} \ldots X_{M}\right)$ is the random vector (spanning the input stochastic space) with known Probability Density Function (PDF) $f_{X}$, and $Y$ is a scalar output which can be expanded as the weighted sum of polynomials (4) [13]:

$$
Y=\mathcal{M}(\boldsymbol{X})=\sum_{\boldsymbol{\alpha} \in \mathbb{N}^{M}} y_{\alpha} \Phi_{\alpha}(\boldsymbol{X})
$$

where $\boldsymbol{\alpha}$ is a multi-index, $\Phi_{a}(\boldsymbol{X})$ form a multivariate polynomial basis in the Hilbert space $L_{P_{x}}^{2}\left(\mathbb{R}^{M}, \mathbb{R}\right)$ with the inner product $\left\langle u, v>=\int_{\mathbb{R}^{M}} u(\boldsymbol{x}) v(\boldsymbol{x}) f_{X}(\boldsymbol{x}) \mathrm{d} \boldsymbol{x}\right.$, and $y_{\alpha}$ are the PCE coefficients. The PCE may also have sparse expression where only desired polynomials are conserved, as in (5):

$$
Y \approx \sum_{\alpha \in \mathcal{A}} y_{\alpha} \Phi_{\alpha}(\boldsymbol{X})
$$

where $\mathcal{A} \subset \mathbb{N}^{M}$ is a subset of index of "dominant polynomials" of total degree less than a prescribed value.

The prediction accuracy of the PCEM will be evaluated and controlled by the cross-validation indicator known as the Leave-One-Out error $\left(e_{L O O}\right)$ [14] defined in (6):

$$
e_{L O O}=\sum_{n=1}^{N}\left[\mathcal{M}\left(\boldsymbol{x}^{(n)}\right)-\mathcal{M}^{P C \backslash n}\left(\boldsymbol{x}^{(n)}\right)\right]^{2} / \sum_{n=1}^{N}\left(Y^{(n)}-\hat{\mu}_{Y}\right)^{2}
$$

where $\mathcal{X}=\left\{\boldsymbol{x}^{(1)}, \ldots, \boldsymbol{x}^{(N)}\right\}^{\mathrm{T}}$ is the Experimental Design (ED) of $N$ realizations of $\boldsymbol{X}$ throughout which the system is pre-evaluated, with responses noted as $\mathcal{Y}=\left\{Y^{(1)}, \ldots, Y^{(N)}\right\}^{\mathrm{T}}$; $\mathcal{M}^{\mathrm{PCIn}}$ is the PCE model derived from the whole set $\{\mathcal{X}, \mathcal{Y}\}$ except $\left\{\boldsymbol{x}^{(n)}, Y^{(n)}\right\}$, and $\hat{\mu}_{Y}$ is the mean of the responses.

Taking the random disturbances as inputs, and the dominant $\mathcal{H}_{q}(f)$ as outputs, we can expand each $\mathcal{H}_{q}(f)$ into the form of (5) to obtain a compact surrogate model (7) for the ATFs:

$$
\mathcal{H}(\hat{\mathbf{r}}, \boldsymbol{X})=j \sum_{q \in I_{d}} \sum_{\boldsymbol{\alpha} \in \mathcal{A}_{q}}(-j)^{\bmod (q, 2)} y_{\alpha}^{q} \Phi_{\alpha}(\boldsymbol{X}) \hat{\boldsymbol{\Psi}}_{\lceil q / 2\rceil}(\hat{\mathbf{r}})
$$

Note that the parameter " $f$ " is omitted in (7) because it can be either set at a fixed value or considered as a component of $\boldsymbol{X}$.

There are a variety of methods for estimating the PCE coefficients. Without trying to be exhaustive, we can mention the Monte-Carlo (MC), the Quadrature Projection (QP), the Ordinary Least Square Error (OLSE), and the Hybrid Least Angle Regression (HLARS) methods [13], etc. Among all these methods, the HLARS has the advantage of being more compact as it conserves only polynomials of the most "correlation-dependent" importance. Moreover, the HLARS is also computationally efficient as thanks to its compact expression, the ED can be designed much tighter, reminding that the cost for the antennas pre-evaluation is proportional to the cardinal of the ED $\left(N_{E D}=\right.$ card(ED)). Additionally, the HLARS can be used in an adaptive way in which the PCE is applied iteratively for increasing polynomial degrees until the expansion accuracy cannot be further improved. In this letter, the adaptive HLARS method is adopted to estimate the PCE coefficients [13].

\section{Statistical Modeling procedure}

The overall modeling of a given antenna undergoing random disturbances $\boldsymbol{X}$ comprises the following steps:

1. Initialize the ED $\mathcal{X}$ and the starting value of $E_{c \_t h}$; impose the RRMSE threshold (RRMSE $\left.E_{t h}\right)$ for the SME truncation and the Leave-One-Out error threshold $\left(e_{\text {LOO_th }}\right)$ for the PCE-HLARS algorithm;

2. Pre-evaluate antenna responses throughout the ED set. Precisely, for each value $\boldsymbol{x}^{(n)}, n=1, \ldots, N_{E D}$, design the corresponding antenna, carry out the EM simulations or measurements and compute the ATF $\mathcal{H}\left(\hat{\mathbf{r}}, \boldsymbol{x}^{(n)}\right)$;

3. Apply the SMEM to each ATF under the energy threshold $E_{c_{-} t h}$ criterion and obtain the dominant index set $I_{d}^{(n)}$;

4. Define the aggregated modes index set $I_{D}=\bigcup_{n=1}^{N_{E D}} I_{d}^{(n)}$;

5. Re-expand all ATFs on modes $\left\{\widehat{\psi}_{[q / 2]}(\hat{\mathbf{r}}), q \in I_{D}\right\}$ and obtain corresponding VSH coefficients $\left\{\mathcal{H}_{q}, q \in I_{D}\right\}$. Note that the steps 4 - 5 are to guarantee that all expansions are based on the same set of modes, whereas the energy criterion $E_{c_{-} t h}$ is still satisfied; 
6. Reconstruct the ATFs from the truncated modes $\left\{\mathcal{H}_{q}\right.$, $\left.\widehat{\psi}_{[q / 2]}(\hat{\mathbf{r}}), q \in I_{D}\right\}$ and assess the error of reconstruction. If $R R M S E \leq R R M S E_{t h}$ is satisfied then pass to step 7, otherwise increase $E_{c_{-} t h}$ and go back to the step 3;

7. Apply the PCE-HLARS method to each $\mathcal{H}_{q}$ and finally get PCE models $\left\{\mathcal{H}_{q}=\sum_{\alpha \in \mathcal{A}_{q}} \mathrm{y}_{\alpha}^{q} \Phi_{\alpha}(\boldsymbol{X}), q \in I_{D}\right\}$;

8. Assess $e_{\mathrm{LOO}}$. If $e_{\mathrm{LOO}} \leq e_{\mathrm{LOO} \text { th }}$ is satisfied for all PCE models, or if $N_{E D}$ exceeds a predefined acceptable value $N_{E D, \max }$ (i.e. too costly to continue) then terminate the modeling procedure; otherwise enrich the ED (i.e. $\left.\mathcal{X}_{\text {new }}=\left[\mathcal{X} ; \mathcal{X}_{\text {add }}\right]\right)$ and go back to the step 2 .

Eventually, a surrogate model relating quantitatively and explicitly the random disturbances $\boldsymbol{X}$ and the ATFs via the whole set of polynomial coefficients $\left\{\mathrm{y}_{a}^{q}, \alpha \in \mathcal{A}_{q}, q \in I_{D}\right\}$ is established, allowing the ATF generation for any value of $\boldsymbol{X}$.

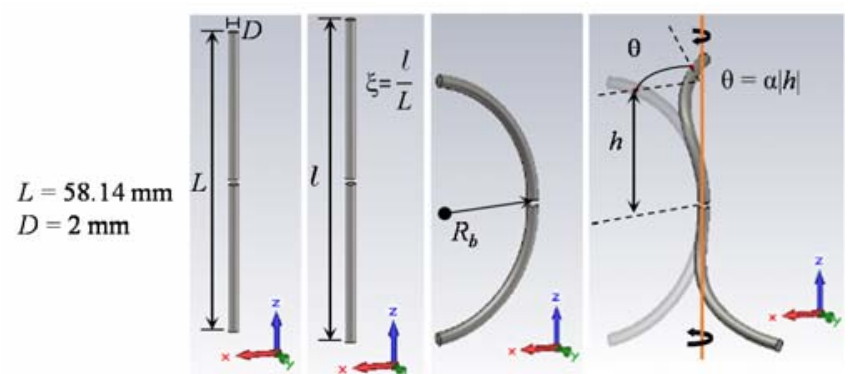

(a)

(b)

(c)

(d)

Fig. 1. Dipole undergoing different deformations: (a) original dipole; (b) stretched dipole; (c) curved dipole (d) curved and twisted dipole.

\section{Application To A Deformable Dipole}

The proposed methodology is, among others, applied to a lossless half-wave wire dipole which is a simple and very well-known canonical antenna (so an ideal "test antenna") to verify its relevance, and assess its accuracy and robustness. Besides, a few results are briefly given for a deformable patch.

\section{A. Design of the deformable dipole}

The original antenna (Fig. 1 (a)) is designed around $2.3 \mathrm{GHz}$ and well matched $\left(\left|\mathrm{S}_{11}\right|<-10 \mathrm{~dB}\right)$ over a frequency band of $[2.1,2.4] \mathrm{GHz}$. The dipole is assumed to be deformable using three independent parameters: stretching (with stretch ratio $\xi$ ), bending (with curvature $\kappa$ or bending radius $R_{b}=1 / \kappa$ ), and torsion (with torsion rate $\alpha$ ), see Fig. 1 (b)-(d). We suppose that the input parameters $(\xi, \kappa, \alpha)$ are uniformly distributed over respectively $[1,1.05],[0,0.1] \mathrm{mm}^{-1}$, and $[0,0.1] \mathrm{rad} \cdot \mathrm{mm}^{-1}$. Fig. 2 shows the combined effects of disturbances on the reflection coefficient $\left|\mathrm{S}_{11}\right|$ and on the radiation patterns (at $f=2.3 \mathrm{GHz}$ ). Compared to the original dipole, variations of more than $15 \mathrm{~dB}$ in $\left|\mathrm{S}_{11}\right|$ and $13.5 \mathrm{~dB}$ in $G_{r}$, i.e. $92.6 \%$ in terms of RRMSE are observed; these large variations justify to resort to an accurate quantitative approach. In the following, the considered frequency is set to $f=2.3 \mathrm{GHz}$.

\section{B. Modeling description and results}

The modeling approach described in section II.C is applied to the dipole. Firstly, the ED is initialized with 100 samples using the Latin-Hypercube-Sampling (LHS) algorithm [15]; the desired thresholds $R R M S E_{\text {th }}$ and $e_{\text {Loo } t \text { th }}$ are fixed at $2 \%$ and $4 \%$; the starting value for $E_{c_{-} t h}$ is set at $80 \%$. Secondly, the pre-evaluation of the dipole is carried out in $\mathrm{CST}^{\circledR}$ MWS (with the FEM solver) to obtain radiation fields which are sampled every $5^{\circ}$ in both elevation and azimuth. The full truncation degree of the SME is set at $N_{\max }=3$ (i.e. $P_{\max }=30$ ), for which the collected energy is proved to be very close to the total energy of the EMF in reality. The "Maximin" technique is used for enriching the initial ED, the principle of which is to add new points sequentially to the ED while maximizing the minimum Euclidean distance between the new points and the existing ones. At last, we use the UQLab, a toolbox for the uncertainty quantification developed by the team of B. Sudret [16], to implement the PCE-HLARS algorithm.

As shown in Fig. 3, while increasing the collected energy threshold $E_{c_{-} \text {th }}$, the RRMSE of the SME decreases gradually but meanwhile the number of selected dominant $\mathcal{H}_{q}$ increases rapidly, which degrades the compactness of the model. The $R R M S E_{\text {th }}$ is ultimately satisfied with $E_{c_{-} \text {th }}=99.9 \%$, with $35(<<$ $\left.2 P_{\max }\right)$ selected dominant $\mathcal{H}_{q}$. Fig. 4 shows the $e_{L O O}$ of the PCE models for the selected dominant $\mathcal{H}_{q}$. As the mode indexing is chosen once, there is no way to guarantee a monotonic decrease of the modal energy with the global index $q$ for all samples in the ED (as energy dominance can vary from one sample to another). It is why $e_{L O O}$ does not increase monotonically with $q$ in Fig. 4 (although of course a less energetic mode is more sensitive to noise). Finally it can be seen that when $N_{E D}$ increases the error decreases for all PCE models and the $e_{L O O \_t h}$ is satisfied for all models for $N_{E D}=260$, for which we get a surrogate model with about 2000 polynomial coefficients.

In order to verify its accuracy and robustness, the constructed model has been used to generate the most deformed dipole (i.e. $(\xi, \kappa, \alpha)=(1.05,0.1,0.1))$ and a comparison of radiation patterns between the initial field, the reconstructed field (with dominant VSH modes), and the generated field (with the proposed surrogate model) is presented in Fig. 5(a). It is noted that the maximal difference in $G_{r}$ is only $0.7 \mathrm{~dB}$ and the RRMSE is less than $8 \%$ in this extreme deformation case; furthermore, the model has been used to generate dipoles for 500 random values of $(\xi, \kappa, \alpha)$, which are compared to their simulated (with $\mathrm{CST}^{\circledR}$ MWS) counterparts, as shown in Fig. 5(b). We see that the RRMSE of the generated antennas remains below $2 \%$ for $88 \%$ of the cases and the extreme error is still less than $10 \%$, which confirms the accuracy and the robustness of the model. It should be noted that there is always a trade-off between the model accuracy and the cost to achieve that precision. One can choose the most appropriate trade-off according to each practical needs. Another conclusion we can draw is that the model also verifies high parsimony. Compared to the initial antennas representation by a huge amount of sampled EMF data $\left(\sim 10^{6}\right)$, the surrogate model requires only a limited number of parameters (i.e. $\sim 10^{3}$ ), reaching a data compression rate of about $10^{3}$. It is actually much higher as input data (parameters or angle variables) can be interpolated as desired. Besides, the modeling was applied to other antennas such as e.g. a microstrip-fed patch with variable length and substrate curvature, thickness and $\varepsilon_{r}$ to show its feasibility for any type of antennas or deformations. For $N_{E D}=280$, a model of $\sim 2600$ PCE coefficients and RRMSE $<15 \%$ was achieved. 


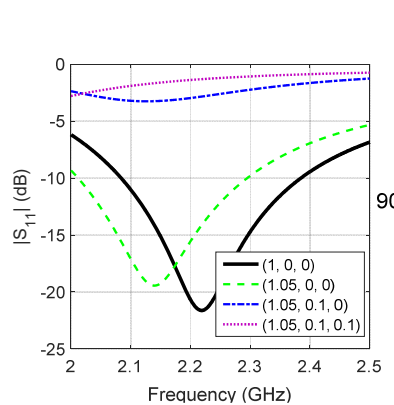

(a)

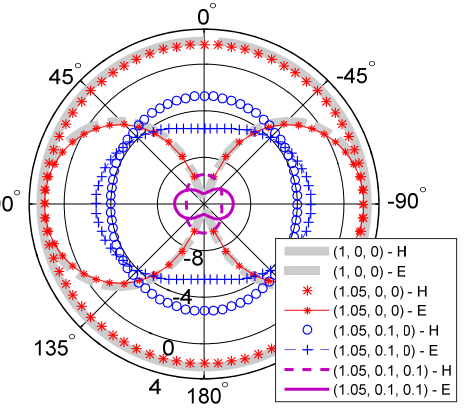

(b)
Fig. 2. Combined effect of stretching, bending, and torsion $(\xi, \kappa, \alpha)$ on the dipole: (a) reflection coefficient $\left|S_{11}\right|$; (b) radiation patterns (in $\mathrm{E} / \mathrm{H}$ plane) at $f=$ $2.3 \mathrm{GHz}$.

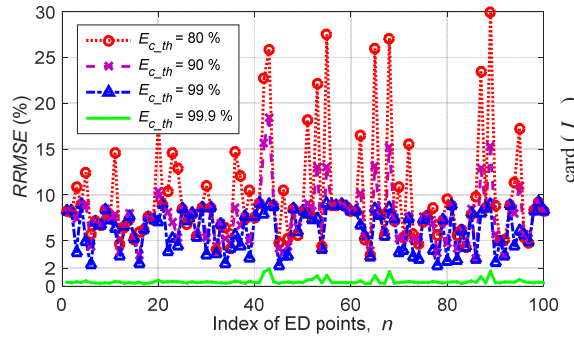

(a)

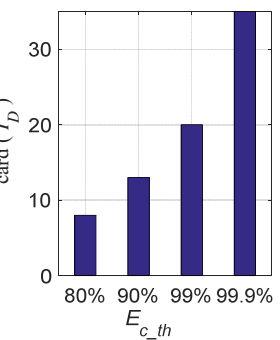

(b)
Fig. 3. (a) RRMSE of reconstructed fields; (b) number of selected dominant $\mathcal{H}_{q}$ : for different collected energy threshold $E_{c-t h}$ for $N_{E D}=100$.

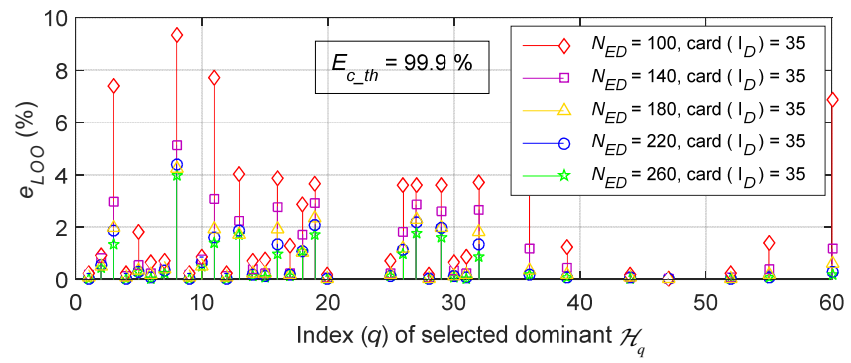

Fig. 4. $e_{L O O}$ of the PCE models for selected dominant $\mathcal{H}_{q}$ for different ED.

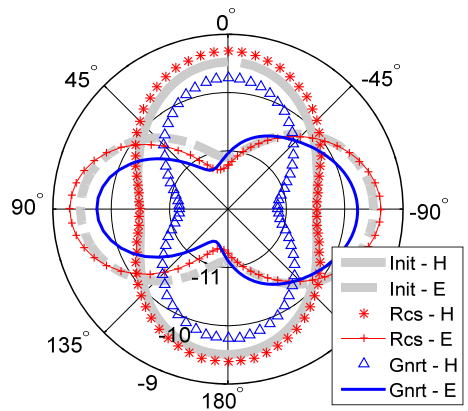

(a)

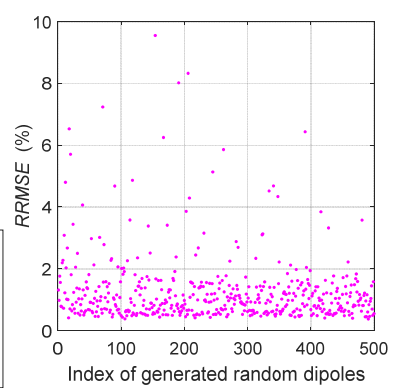

(b)
Fig. 5. (a) Radiation patterns (in E/H plane) of the initial, reconstructed and generated fields for the most deformed case at $f=2.3 \mathrm{GHz}$; (b) RRMSE of 500 generated dipoles for random values of $(\xi, \kappa, \alpha)$.

\section{CONCLUSION}

The proposed methodology allows to develop compact and precise surrogate models of the FF radiated by "random antennas" with a minimized cost. This methodology has been applied to the modeling of a deformable dipole to prove its feasibility, efficiency and high accuracy. The derived model is computationally costless for generating deformable dipoles usable as surrogate models in various types of analyses requiring the complete field knowledge (including polarization and phase). The proposed modeling methodology is applicable to any other types of random disturbances or antennas. Future and already on-going work will address more realistic applications considering e.g. flexible printed antennas for terminals or sensors and/or perturbations due to the nearby surrounding environment, including the modeling of the frequency behavior $\left(S_{11}(f)\right.$, etc. $)$.

\section{ACKNOWLEDGMENT}

The authors would like to thank Pr. B. Sudret and the UQLab team for providing PCE-HLARS freewares for academics, and Dr. J. Wiart for fruitful discussions and useful advices.

\section{REFERENCES}

[1] N. H. M. Rais, P. J. Soh, F. Malek, S. Ahmad, N. B. M. Hashim, and P. S. Hall, "A review of wearable antenna," in LAPC, Loughborough, UK, 2009.

[2] M.A Mellah, A. Sibille, C. Roblin, M. Nedil, and T.A Denidni, "Statistical Modeling of the "Antenna-Head" Interaction," IEEE Antenn. Wireless Propag. Lett., vol. 10, pp. 454-457, 2011.

[3] E. F. Sundarsingh, M. Kanagasabai, and V. S. Ramalingam, "Statistical analysis on the bandwidth of a dual frequency textile antenna," IET Microwaves, Antennas \& Propagation, vol. 9, no. 15, pp. 1683-1690, Dec. 2015.

[4] F. Boeykens, L. Vallozzi, and H. Rogier, "Cylindrical bending of Deformable textile rectangular patch antennas," International Journal of Antennas and Propagation, vol. 2012, pp. 1-11, 2012.

[5] P. Kersaudy, S. Mostarshedi, B. Sudret, O. Picon, and J. Wiart, "Stochastic analysis of scattered field by building facades using polynomial chaos," IEEE Trans. Antennas Propag., vol. 62, no. 12, pp. 6382-6393, Dec. 2014.

[6] A. Sibille, C. Roblin, S. Bories, and A. C. Lepage, "A Channel-Based Statistical Approach to Antenna Performance in UWB Communications," IEEE Trans. Antennas Propag., vol. 54, n 11, pp. 3207-3214, Nov. 2006.

[7] J. E. Hansen (Ed.), Spherical near-field antenna measurements, IEEE Electromagnetic waves series 26, London, UK: Peter Peregrinus, 1988.

[8] C. E. Baum, "On the singularity expansion method for the solution of Electromagnetic interaction problems," Interaction Notes, Note 88, 1971.

[9] Ch. Roblin, "Ultra compressed parametric modelling of UWB antenna measurements," in EuCAP, Nice, France, 2006.

[10] Z. Mhanna, A. Sibille, M. A. Yousuf, and Ch. Roblin, "Parametric statistical modeling of power gain patterns for RFID backscattering channels," in EuCAP, Prague, 2012.

[11] Ch. Roblin, S. Bories and A. Sibille, "Characterization tools of antennasin the Time Domain," in IWUWBS, Oulu, June 2003.

[12] Jensen, F. and Frandsen, A., "On the number of modes in spherical wave expansion," in Proc. 26th AMTA, Stone Mountain Park, GA, pp. 489-94, Oct. 2004.

[13] G. Blatman and B. Sudret, "Adaptive sparse polynomial chaos expansion based on least angle regression," Journal of Computational Physics, vol. 230, no. 6, pp. 2345-2367, Mar. 2011.

[14] A. M. Molinaro, R. Simon, and R. M. Pfeiffer, "Prediction error estimation: A comparison of resampling methods," Bioinformatics, vol. 21, no. 15, pp. 3301-3307, May 2005.

[15] M. D. McKay, R. J. Beckman, and W. J. Conover, "A comparison of Three methods for selecting values of input variables in the analysis of output from a computer code," Technometrics, vol. 21, no. 2, p. 239, May 1979.

[16] S. Marelli and B. Sudret, UQLab user manual - Polynomial Chaos Expansions, Report UQLab-V0.9-104, Chair of Risk, Safety \& Uncertainty Quantification, ETH Zurich, 2015. 